\title{
Pigeons produce aerodynamic torques through changes in wing trajectory during low speed aerial turns
}

\section{Citation}

Ros, I. G., M. A. Badger, A. N. Pierson, L. C. Bassman, and A. A. Biewener. 2014. “Pigeons Produce Aerodynamic Torques through Changes in Wing Trajectory During Low Speed Aerial Turns." Journal of Experimental Biology 218 (3) (December 1): 480-490. doi:10.1242/jeb.104141.

\section{Published Version}

doi:10.1242/jeb.104141

\section{Permanent link}

http://nrs.harvard.edu/urn-3:HUL.InstRepos:14117004

\section{Terms of Use}

This article was downloaded from Harvard University's DASH repository, and is made available under the terms and conditions applicable to Open Access Policy Articles, as set forth at http:// nrs.harvard.edu/urn-3:HUL.InstRepos:dash.current.terms-of-use\#OAP

\section{Share Your Story}

The Harvard community has made this article openly available.

Please share how this access benefits you. Submit a story.

\section{Accessibility}




\section{Title}

2 Pigeons produce aerodynamic torques through changes in wing trajectory during low speed

3 aerial turns

4

\section{Short Title}

6 Aerodynamic torque generation in turning pigeons

8 Authors. Ivo G. Ros ${ }^{1}$, Marc A. Badger ${ }^{2}$, Alyssa N. Pierson ${ }^{3}$, Lori C. Bassman ${ }^{3}$ and Andrew A.

9 Biewener $^{1}$

11 Author affiliation. 'Harvard University, Department of Organismic and Evolutionary Biology,

12 Concord Field Station, 100 Old Causeway Road, Bedford, MA 01730, USA

13 '2University of California, Berkeley, Department of Integrative Biology, 3060 VLSB

14 \#3140, Berkeley, CA 94720, USA

$15{ }^{3}$ Harvey Mudd College, Department of Engineering, 301 Platt Blvd., Claremont, CA 91711, 16 USA

\section{Keywords}

19 Pigeon; Turning; Aerodynamics; Dynamics Modeling; Inertia

\section{Summary}

The complexity of low speed maneuvering flight is apparent from the combination of two

23 critical aspects of this behavior: high power and precise control. To understand how such control

24 is achieved we examined the underlying kinematics and resulting aerodynamic mechanisms of

25 low speed turning flight in the pigeon (Columba livia). Three birds were trained to perform 90-

26 degree level turns in a stereotypical fashion and detailed three-dimensional (3D) kinematics were

27 recorded at high speeds. Applying the angular momentum principle, we used mechanical

28 modeling based on time-varying 3D inertia properties of individual sections of the pigeon's body

29 to separate angular accelerations of the torso based on aerodynamics from those based on inertial

30 effects. Directly measured angular accelerations of the torso were predicted by aerodynamic

31 torques, justifying inferences of aerodynamic torque generation based on inside wing versus 
32 outside wing kinematics. Surprisingly, contralateral asymmetries in wing speed did not appear to

33 underlie the 90-degree aerial turns, nor did contralateral differences in wing area, angle of attack,

34 wingbeat amplitude, or timing. Instead, torso angular accelerations into the turn were associated

35 with the outside wing sweeping more anteriorly compared to a more laterally directed inside

36 wing. In addition to moving through a relatively more retracted path, the inside wing was also

37 more strongly pronated about its long axis compared with the outside wing, offsetting any

38 difference in aerodynamic angle of attack that might arise from the observed asymmetry in wing

39 trajectories. Therefore, to generate roll and pitch torques into the turn, pigeons simply reorient

40 their wing trajectories toward the desired flight direction. As a result, by acting above the center

41 of mass, the net aerodynamic force produced by the wings is directed inward, generating the

42 necessary torques for turning.

\section{Introduction}

To gain insight into the control of flight maneuvers, an understanding of the mechanics and aerodynamics involved in turning is needed. However, to date turning flight has received

47 little attention compared with steady forward flight.

Mechanistically, the task of turning consists of two conceptually separate components: 1) re-direction of the animal's flight path and 2) reorientation of the animal's body.

Re-direction of the flight path (involving changes in translational movement) requires

51 centripetal force production. Slowly flying vertebrates, including horseshoe bats, pigeons, fruit

52 bats and cockatoos, all bank to orient the net aerodynamic force into the turn, analogous to how

53 fixed-wing aircraft turn (Aldridge, 1989; Warrick and Dial, 1998; Hedrick and Biewener, 2007;

54 Iriarte-Diaz and Swartz, 2008; Ros et al., 2011).

55 Reorientation of the torso (involving rotational movement) in flight can be achieved 56 using two physical principles: (i) aerodynamic forces that induce torques about the center of 57 mass (CM), and (ii) inertia-based changes in angular momentum of body segments that result in 58 net changes in body orientation (Frolich, 1980; Hedrick et al., 2007). 3D analysis of the mass

59 distributions and time-varying configurations of the head, torso and wings can be used to 60 determine both aerodynamics- and inertia-based maneuvering in flying birds. 
Irrespective of the nature of the torque generating mechanisms, the initiation and active

62 control of maneuvering flight requires asymmetries in force production by the animal's

63 locomotor system. Thus, in order to identify underlying neuromuscular control mechanisms of

64 turning flight, such mechanisms must be resolved on a fine timescale (within wingbeats).

65 Prior work has shown that pigeons and cockatoos redirect net aerodynamic forces through

66 bilateral asymmetries in both downstroke velocity and angular momentum of the two wings

67 (Warrick and Dial, 1998; Hedrick et al. 2007). Furthermore, pigeons maneuvering through a

68 course with multiple turns generate small, sequential, pectoralis muscle force asymmetries to

69 bank and navigate through the course (Warrick et al. 1998).

70 Recently, we (Ros et al., 2011) have shown that reorientations of the pigeon's torso are

71 integral to low speed turning flight. Furthermore, torso rotations underlying flight path changes

72 (constituting torque generation for steering) were found to occur subsequent to readily

73 observable head rotations (head saccades) during the downstroke of the same wingbeat cycle

74 (Ros and Biewener, in review). These torso rotations must be produced by torques resulting from

75 asymmetrical force production by the wings and/or tail. Here we seek to identify the key

76 mechanism underlying low speed turning in pigeons, by testing whether the relevant turning

77 torques are of an aerodynamic or inertial nature, and by investigating how contralateral wing

78 movement asymmetries underlie torque generation. Because wingbeats containing head saccades

79 early in the turn likely display the strongest torque generation (Ros and Biewener, in review),

80 eighteen specific wingbeats were selected for analysis (see Materials and Methods for details).

81 Based on previous findings in pigeons, cockatoos and bats (Warrick and Dial, 1998; Hedrick et

82 al. 2007; Iriarte-Diaz et al., 2011), we expected inertial and aerodynamic effects to act

83 synergistically in rotating the torso, because both aerodynamics-based and inertia-based torques

84 would act in the opposite direction of a faster moving wing. We hypothesized further a greater

85 influence of aerodynamic torques, generated through contralateral asymmetries in wing velocity

86 and aerodynamic angle of attack (Warrick and Dial, 1998; Hedrick et al. 2007). 
To test these hypotheses, we record detailed kinematics of three pigeons negotiating a

90 level 90-degree turn (Fig. 1). By merging morphological mass-distribution measurements of the

91 pigeon's wings, head, torso and tail with these positional data (Fig. 2), we generate a full three-

92 dimensional (3D) dynamics model, which can separate aerodynamics-based from inertia-based

93 angular accelerations of the turning pigeon's torso based on application of the conservation of

94 angular momentum principle. A determination of the timing and magnitude of both

95 aerodynamics- and inertia-based torso angular accelerations is expected to provide a more

96 accurate description of the rotational component of the maneuver, as well as to allow for more a

97 detailed identification of the wing and tail kinematics underlying these torso angular

98 accelerations.

99

100

101

102

103

104

105

106

107

108

109

110

111

112

113

114

115

\section{Results}

The three pigeons negotiated the $90^{\circ}$ level turns at a CM speed of $3.3 \pm 0.2 \mathrm{~m} \mathrm{~s}^{-1}$ and a wingbeat frequency of $8.3 \pm 0.3 \mathrm{~Hz}$. In terms of mass distribution, each wing (distal to the shoulder) comprised $6.3 \pm 0.7 \%$ and the head $3.9 \pm 0.3 \%$ of the bird's body mass $(319 \pm 33 \mathrm{~g}$; Table 1).

\section{Torso reorientations about all three anatomical axes}

The low-speed, level turns were predominantly driven by roll and yaw rotations of the pigeons (Fig. 3). Only pitch fluctuated with consistent periodicity within the wingbeat cycle. Torso orientations about all three anatomical axes fluctuated within wingbeats, independent of changes in torso orientation across wingbeats. These latter net wingbeat reorientations about the roll and yaw torso axes, summed throughout the turn, comprised the majority of the torso reorientations across wingbeats (Table 2). The birds consistently yawed into the turn, whereas roll was initially directed into the turn and later directed outward from the turn. Net wingbeat pitch reorientations were generally directed upward (head-up). 
117 The angular momentum principle (Pratab and Ruina, 2009; Mitiguy, 2014) allowed for 118 calculation of inertia-based angular accelerations of the torso, and, indirectly, for calculation of 119 the aerodynamics-based equivalent. Using wing-segment masses and moments of inertia for the 120 torso (which includes the tail) and head, the angular momentum principle was applied to the 121 time-varying 3D marker positions. In brief, two separate main modeling steps were performed:

122 (i) aerodynamics-based torques were estimated directly from the 3D positional data and 123 morphological mass distributions; (ii) inertia-based torso angular acceleration were estimated 124 based on the hypothetical case where the aerodynamic forces were assumed to be absent. The 125 central angular momentum, i.e. the combined angular momentum of the entire body about the 126 collective CM, can be calculated from time-varying mass distributions data (see Eqn 1). The 127 aerodynamic torque follows directly from the rate change of the central angular momentum; 128 whereas, by hypothetically equating the aerodynamic torque to zero the inertial angular effect of 129 measured wing motions on the pigeon's torso can be inferred (see Materials and Methods section 130 for details).

131 We found that aerodynamic torques, and not inertia-based angular momentum exchanges 132 between the wings and the rest of the bird (torso, head and tail) predicted observed torso angular 133 roll and pitch accelerations (Fig. 4). Estimated angular accelerations of the torso based on 134 aerodynamics correlated positively with measured accelerations about each of the principal torso 135 axes. These aerodynamics-based estimates most reliably predicted pitch rotations $\left(\mathrm{p}<0.01, \mathrm{R}^{2}=\right.$ 136 0.89), with significant torso acceleration correlations about both roll $\left(\mathrm{p}<0.01, \mathrm{R}^{2}=0.72\right)$ and yaw $137\left(\mathrm{p}<0.01, \mathrm{R}^{2}=0.62\right)$ of similar strength. The range of estimated yaw accelerations was smallest, 138 the only axis about which inertia-based estimates also predicted observed torso accelerations 139 ( $\mathrm{p}<0.01, \mathrm{R}^{2}=0.41$; Fig 4). During the turning wingbeats selected for analysis of torque 140 generating mechanisms, roll rotations were initiated early and arrested late in the downstroke, 141 with a peak in angular velocity directed into the turn at mid downstroke (Fig. 5A \& B). Variable 142 roll velocities were of much smaller magnitude during the upstroke. Torso pitch velocities and 143 accelerations consistently varied with wingbeat phase: head-up (positive) pitch acceleration 144 peaked near the middle of upstroke and approximately two-thirds into downstroke, coincident 145 with negative roll acceleration (out of the turn); whereas head-down (negative) pitch acceleration 
146 peaked near the first third of downstroke, coincident with the peak in positive roll acceleration.

147 These pitch patterns were consistent for all wingbeats analyzed throughout the turn, and

148 consistent with aerodynamic torques (Fig. 8A \& B). In contrast, torso roll and yaw aerodynamic

149 torques and resulting angular movements (not shown) were more variable throughout the turn.

150 Yaw velocity was consistently positive (directed into the turn) over the full wingbeat cycle (Fig.

$1515 \mathrm{~A}$ ), with a moderate deceleration phase near the end of downstroke (Fig. 5B).

\section{Model sensitivity and data validation}

154 Approaches containing double derivatives of positional data are sensitive to measuring errors, 155 warranting sensitivity tests of our dynamics model. Artificially varying the wing length by \pm $15610 \%$ and changing the mass of the hand wings (the point masses distal to the wrist) by $10 \%$ of 157 the wing mass, did not change statistical trends between inertia- or aerodynamics-based and 158 observed angular torso accelerations. Artificially increasing the wing lengths or hand wing 159 masses resulted in a slightly reduced regression model fit between aerodynamics-based estimates 160 and observed torso angular accelerations $\left(\mathrm{R}^{2}\right.$ values for roll $(0.72)$, pitch $(0.89)$ and yaw $(0.62)$ 161 decreased to $0.69,0.87$ and 0.56 for wing lengths, and decreased to or stayed equal at $0.64,0.89$ 162 and 0.38 for hand wing masses, respectively). Artificially decreasing wing lengths or hand wing 163 masses lead to similar changes, but in the opposite direction. The sensitivity tests most strongly

164 affected torso acceleration estimates about the yaw axis, about which inertia-based estimates also 165 predicted observed torso angular accelerations, albeit with a lower slope (0.34 vs 0.66) and 166 regression model fit $\left(\mathrm{R}^{2}=0.41\right.$ vs 0.62$)$ than aerodynamics-based estimates. Additionally, the 167 marker-based torso angular accelerations compared well to measurements obtained from a back168 mounted inertial measurement unit (IMU) in pigeons maneuvering at similar strengths and flight 169 speeds (Lin et al., in prep). Besides similarities in trace shape within wingbeat cycles, peak torso 170 angular velocities and accelerations reached approximately $900 \mathrm{deg} \mathrm{s}$ and $7 * 10^{4} \mathrm{deg} \mathrm{s}^{-2}$,

171 respectively, for both kinematics derived and IMU-based values. Although the IMU recordings

172 were of pigeons during different maneuvers and should not be directly compared, their similar 173 patterns are affirmative and support the sensitivity tests in illustrating the robustness of our 174 finding of aerodynamics-based torque generation. 
Turning wingbeats selected for analysis of torque generating mechanisms were

179 characterized by differentially swept wing trajectories, or paths of wing motion relative to the

180 torso (Fig. 6), accompanied by contra-lateral differences in hand-wing axial (pronation-

181 supination) orientation (Fig. 7A). Corresponding with the peak in roll acceleration observed early

182 in downstroke (Fig 5B), the outside wing was initially positioned and swept more anteriorly

183 compared to a more laterally directed inside wing trajectory (Fig 6). In concert with the contra-

184 lateral asymmetry in wing trajectories, the inside wing was more strongly pronated, or rotated

185 forward (leading-edge down) about the wing's long axis during the first half of the downstroke

186 (Fig. 7A). This contra-lateral difference in pronation was reversed in the second half of the

187 downstroke, through supination of the inside wing and pronation of the outside wing near the

188 middle of the downstroke (Fig. 7A). As the pigeon's torso rolled into the turn in the downstroke

189 (Fig. 5A), the inside wing also acquired a higher speed (Fig. 7B) and became oriented more

190 laterally (Fig. 7C) compared with the outside wing. speed turning flight were determined using segment masses, time-varying 3D inertia properties

195 and torso and wing configurations. Using this approach, we show that measured torso angular 196 accelerations correlate strongly with observed aerodynamic torques, justifying a comparison of

197 inside wing versus outside wing kinematic proxies for aerodynamic torque generating 198 mechanisms. In comparison to previous findings (Warrick and Dial, 1998), contralateral

199 asymmetries in wing speed do not appear to underlie the aerial turns we analyzed, and 200 surprisingly, nor do contralateral differences in wing area, angle of attack, wingbeat amplitude, 201 or timing. Instead, to generate roll and pitch torques into the turn, the pigeons reorient their wing

202 trajectories toward the desired direction while rotating their hand-wings such that similar 203 aerodynamic angles of attack are maintained between the inside and outside wing (Figs. 6 \& 7).

204 As a result, the aerodynamic force is directed into the turn and, by acting above the center of 205 mass, generates the necessary turning torques (Fig. 8). 
Our results show that pigeons use a complex combination of roll, pitch and yaw torso rotations to make level turns, with the largest contribution resulting from reorientations about the antero-posterior axis of the torso (Fig. 2; Table 2). These roll rotations serve to redirect the net aerodynamic force, producing changes in the flight path (Ros et al., 2011): during the first half of the turn pigeons roll into the turn, whereas during the second half they roll out. The observed roll

211 patterns may also enable changes in torso pitch to contribute to the turn. Due to the bird's banked 212 orientation while turning (Warrick and Dial, 1998; Hedrick and Biewener, 2007; Ros et al.,

213 2011), head-up pitch reorientations can contribute to reorienting the bird's body into the desired

214 direction. However, neither the summed net wingbeat yaw or pitch reorientations fully matched

215 the 90 degrees of the turn (Table 2), illustrating the need for a composite of whole-body rotation

216 components to simultaneously meet the translational (flight path changes and gravitational

217 support) and rotational (acquisition of the desired new flight orientation) requirements of the 218 turn.

The consistent patterns of aerodynamics-based pitch acceleration and torque observed

221 throughout the wingbeat cycle, strongly suggest that the center of pressure of the resultant 222 aerodynamic force is positioned dorsal to the bird's CM during the first half of downstroke, and 223 ventral and anterior to the CM near the second half of downstroke, through the middle of 224 upstroke. These inferences agree with previous findings that aerodynamic forces act dorsal to the 225 center of mass during the downstroke of the Japanese White-eye (Su et al., 2011) and 226 corroborate the active aerodynamics of the pigeon's tip-reversal upstroke (Crandell and 227 Tobalske, 2011; Ros et al., 2011).

228 Contralateral differences in wing trajectory and hand-wing pronation (Figs. 6 \& 7A) 229 coincide with roll accelerations observed early in the downstroke that are directed towards the 230 inside wing (Fig. 5B). Consequently, these kinematic asymmetries of the wings likely indicate 231 the aerodynamic mechanism used to generate the necessary steering torque. Torso accelerations 232 that increase the central angular momentum of the bird require differential activation of 233 contralateral flight muscles. Compared to Warrick and Dial (1998), we find here that these 234 'active' torso angular accelerations do not correlate with contralateral asymmetries in wing speed 235 relative to the torso (relevant from a control perspective), or in the inertial frame (relevant to 
236 aerodynamics). Nor were con,tralateral differences in wing length (which may serve as a proxy

237 for wing area), aerodynamic angle of attack, wingbeat amplitude, or timing found to correlate

238 with observed patterns of torso angular acceleration. Instead, pigeons appear to reorient the

239 flapping sweep of their wing trajectories toward the desired direction, while preventing

240 aerodynamic angle of attack asymmetries through hand-wing rotations. These differences in

241 wing trajectories between the inside and outside wing likely redirect the net aerodynamic force

242 into the turn (Fig. 8D). Assuming that this aerodynamic force acts dorsal to the bird's CM, the

243 redirected aerodynamic force would generate the observed roll towards the inside wing, in

244 addition to producing head-down pitch (Fig. 8C \& D).

245 Given broad similarities in the avian body plan and the fairly simple nature of the

246 aerodynamic torque generating mechanism described here, we believe it is likely that other bird

247 species use the same mechanism to generate aerodynamic torques during slow turning flight. For

248 example, cockatoos turning at low speeds displayed contralateral differences in wing kinematics

249 similar to those described here, even though these kinematic differences in the cockatoos did not

250 relate significantly to roll accelerations (Hedrick and Biewener, 2007). It is likely that this

251 reflects the greater kinematic resolution of the current study and recognition that redirection of

252 aerodynamic force occurs at key points of turning that follow head saccades (Ros and Biewener,

253 in review). Asymmetries in wing trajectory and pronation were also found previously in pigeons

254 (Warrick and Dial, 1998). However, technical limitations prevented measurements of pronation

255 angles throughout wingbeat cycles and, thus, linking these asymmetries to torque generation. In

256 the current study, the eighteen turning wingbeats selected for analysis generally occurred early in

257 the turn where the strongest trajectory changes occur (Ros et al., 2011), although the described

258 torque generating mechanism can be expected to be used throughout turns when trajectory

259 changes are needed.

260 The observed contralateral differences in wing trajectory and hand-wing pronation further

261 corroborate the idea that antebrachial musculature is involved in low speed flight maneuvers

262 (Bilo, 1985; 1994; Dial, 1992). Our results, however, do not exclude the potential role of

263 differential force production between contralateral pectoralis muscles of the inside and outside

264 wing (Warrick et al., 1998) or corresponding differential wing speeds (Warrick and Dial, 1998)

265 as additional or complementary torque generating mechanisms. It is possible that our selection of 
266 key turning wingbeats related to flight trajectory changes excluded other wingbeats in which 267 wing speed asymmetries may contribute to torque production for turning. It is also likely that our 268 improved resolution of temporal and spatial kinematics allow us improved accuracy for wing 269 kinematics relative to movements of the bird's torso. Based on our analysis, we found that 270 differences in wing speed occur near the middle and end of the downstroke (Fig. 7B), when roll 271 velocities are substantial and are subsequently arrested (Fig. 5A). Because rotation of the torso 272 increases the speed of the wing on the side of the bird that moves with torso roll (the inside 273 wing), such a passive rotational damping mechanism (Hedrick et al., 2009) may help to arrest the 274 bird's roll velocity near the end of the downstroke. By projecting more laterally during the 275 second half of downstroke (Figs. $6 \&$ 7C), the inside wing's moment arm for aerodynamic force 276 about the roll axis may also be increased, further enhancing rotational damping. To the extent 277 that torso rotations are passively damped by these mechanisms, such torso rotations would 278 mitigate the need for differential activation of contralateral wing muscles to arrest existing torso 279 angular rotations.

280 Our mechanical modeling approach depends on second time-derivatives of estimated 281 mass distributions, which renders it sensitive to the propagation of measuring errors. Therefore, 282 we limit our conclusions based on the dynamics model to correlative patterns that indicate the 283 nature of the torques underlying these turns (inertia or aerodynamics; Fig. 4) and to consistent 284 aerodynamic pitch torques averaged over many turning wingbeats (Fig. 8A, B) that aid in 285 interpreting the aerodynamic torque generating mechanism used to turn (Fig. 8C, D). More 286 specific inferences, such as time-varying torque patterns within individual wingbeats, await 287 further validation of the modeling and error analyses.

288 By examining the activation and strain patterns of flight muscles throughout aerial turns 289 we plan to further explore the neuromuscular components of flight control. Differential 290 activation of distal wing muscles has been found to relate to steering in simulated flight in 291 pigeons (Bilo and Bilo, 1983). Additionally, the extensor metacarpus radialis, an antebrachial 292 hand wing extensor, was found to predict lower frequency components of turning (Hedrick and 293 Biewener, 2007). Differential activation of inside versus outside wing muscles that are likely to 294 control wing trajectory, such as the scapulohumeralis caudalis and pectoralis pars

295 sternobrachialis (Dial et al. 1991, Baier et al. 2013), and hand wing pronation, such as the 
extensor metacarpi radialis and the ulnometacarpalis ventralis (Vasquez, 1995), may also play

297 key roles in turning flight.

\section{Marker placement and $3 D$ positional reconstruction}

Using the eleven synchronized high-speed cameras, time-varying positions of $10 \mathrm{~mm}$ diameter torso and tail markers, as well as $4 \mathrm{~mm}$ diameter polystyrene wing markers were collected within a calibrated $1.8 \mathrm{~m}^{3}$ cubic volume that encompassed the turn. Trials accepted for analysis (> 90\% of trials recorded) were those in which the birds $(i)$ did not contact the netting and (ii) maintained a turning flight path relative to global horizontal of $<5^{\circ}$. The markers were placed at 16 anatomical locations (see Ros et al., 2011; Fig 2A): dorsum at the second thoracic vertebra; left and right rump (4-cm lateral to the vertebral column over the synsacrum); center of head; left and right wing roots; left and right wrists; tip of left and right fifth primary feathers; 
$67 \%$ of the length of left and right ninth primary feathers; $67 \%$ along the length of left and right

327 outer tail feathers; left and right tip of the innermost secondary feathers. Elbow position was

328 determined trigonometrically based on two lengths and three positions: brachial and antebrachial

329 segment lengths and wing root, wrist, and tip of the innermost secondary feather positions. The

330 maximum mass added to a bird by the markers, including elastic tape on the torso, was $14 \mathrm{~g}$, or

$3314 \%$ of the body mass (BM).

332 The 3D marker positions were reconstructed based on the volumetric calibration, using

333 freely available digitization software (Hedrick, 2008). Calculations were performed in

334 Mathematica (Wolfram Research, Champaign, IL, USA) and Matlab (Mathworks Inc., Natick,

335 MA, USA) using custom-written scripts. Positional data were filtered with a fourth-order

336 Butterworth filter using a low-pass cutoff frequency three times the wingbeat frequency. Cutoff

337 frequency was determined by residual analysis (Winter, 2005). To avoid poor performance of the

338 Butterworth filter near the tails of time series (Walker, 1998), data from 15 frames on either end

339 of each flight trial were omitted post-filtering. The aerodynamic torque filtered with the low-pass

340 butterworth correlated strongly with a quintic spline $\left(\mathrm{R}^{2}=0.98\right)$.

341 Correlations between observed and estimated torso angular accelerations, based on one

342 (peak) value per wingbeat, were tested with standard least squares regression models corrected

343 for trial number, wingbeat number (relative to the geometrical center of the flight corridor, after

344 Hedrick et al., 2007) and individual effects (JMP, SAS Institute, Cary, NC). Unless noted

345 otherwise, results were expressed as mean \pm s.d.

\section{Torso reorientations throughout the turn}

348 Wingbeats were partitioned into upstroke and downstroke phases based on reversal of the 349 major bending direction of the primary feathers. This reversal coincided with the instant the 350 primary feather markers moved laterally relative to the torso, in both ventral (start of upstroke) 351 and dorsal (start of downstroke) positions. Net wingbeat torso rotations were defined as the 352 resulting changes in torso orientation over entire wingbeat periods and were calculated about 353 each of the torso axes. For each of two left and two right turns per individual, at least five 354 sequential upstroke-downstroke wingbeats were analyzed (22, 25 and 28 wingbeats for the three 355 birds), over which net wingbeat torso rotations about each axis were accumulated over the course 
356 of the $90^{\circ}$ turn (Table 2). The analyzed spatial section of the flight corridor was kept consistent

357 across individuals, with the measured change in flight path through the turn averaging to $90.0^{\circ} \pm$

$3582.1^{\circ}$ across individuals.

The angular momentum principle states that the rate of change of the angular momentum

362 of a system, e.g. an entire bird, about its center of mass is equal to the sum of all torques (used

363 here to mean moments of force about the mass center) on the system (Mitiguy, 2014). For the

364 body plan of a pigeon, with many spatial degrees of freedom, the central angular momentum can

365 be calculated through summation of the angular momentum of each body segment about the

366 collective, or system, center of mass $\left(S_{\mathrm{CM}}\right), S_{\mathrm{CM}}$ can move relative to the torso, due primarily to 367 varying wing and head positions.

368 To calculate the central angular momentum, a mass-distribution model was created that 369 treated the torso/tail (modeled as one object) and head as rigid objects, and modeled each wing 370 as a series of point masses $P_{i}$ positioned along the wing segments (Fig. 2; Table 1). Here, the 371 head was assumed to remain in a fixed orientation with respect to the torso and the mass of the

372 tail was included in the torso, ignoring inertial effects due to independent head or tail movement 373 relative to the torso. The wing segments were allocated based on wing anatomy (Fig. 2A). The 374 moments of inertia about the antero-posterior (roll), medio-lateral (pitch), and dorso-ventral 375 (yaw) axes of the head and torso (including the tail) (Table 3) were calculated from the angular 376 swing periods during two-point suspensions (see Newman and Searle, 1957 and Alexander, 377 1968). Additionally, the centers of mass of the head and torso were determined by multiple 378 single-point suspensions as the intersection of the direction of gravity. Only the wing markers 379 were included in the morphometrics.

380 The angular momentum principle formulated with respect to $S_{C M}$ states that angular 381 momentum changes about the center of mass can only be achieved by external torques. Gravity 382 produces a resultant force that acts through $S_{C M}$ and thus does not apply a torque about $S_{C M}$.

383 Aerodynamic forces are the only source of external torques about $S_{C M}$, and therefore the rate of 384 change of the central angular momentum in the inertial reference frame $N$ for the pigeon system $385 S\left(\frac{{ }^{N} d^{N} \vec{H}^{S / S_{\mathrm{CM}}}}{\mathrm{dt}}\right.$; Eqn 1$)$ is equivalent to the aerodynamic torque acting about $S_{\mathrm{CM}}$. 
The expanded central angular momentum principle is given by (see Appendix for derivation):

$$
\frac{{ }^{N} d^{N} \vec{H}^{S / S_{\mathrm{CM}}}}{\mathrm{dt}}=
$$

$$
\left(\sum_{i=1}^{n}\left(m^{P_{i}} *\left[\left(\vec{r}^{P_{i} / \mathrm{T}_{\mathrm{CM}}}-A\right)(D+C+B)\right]\right)+\left(\underline{I}^{\mathrm{T} / \mathrm{T}_{\mathrm{CM}} \cdot N} \vec{\alpha}^{\mathrm{T}}\right)+\left(m^{\mathrm{T}} * D A\right) ;(\text { Eqn } 1)\right.
$$

with:

$$
A=\frac{1}{m^{s}} *\left(\sum_{i=1}^{n}\left(m^{P_{i}} * \vec{r}^{P_{i} / \mathrm{T}_{\mathrm{CM}}}\right)\right)
$$

$D=-\frac{1}{m^{S}} *\left(\sum_{i=1}^{n}\left(m^{P_{i}} *(B+C)\right)\right)$,

for which

$397 m^{P_{i}}=$ wing point mass $P_{i}$;

$398 m^{S}=$ mass of the system $S$ (entire bird);

$399 \underline{I}^{\mathrm{T} / \mathrm{T}_{\mathrm{CM}}}=$ inertia dyadic of the torso $T$ about the center of mass of the torso;

$400 \quad \vec{r}^{P_{i}} / \mathrm{T}_{\mathrm{CM}}=$ vector from the torso center of mass to $P_{i}$;

$401 \mathrm{~T}_{\vec{v}} P_{i}=$ velocity of $P_{i}$ in the reference frame of the torso;

$402{ }^{N} \vec{\omega}^{\mathrm{T}}=$ angular velocity of the torso in the inertial frame;

$403{ }^{\mathrm{T}} \vec{a}^{P_{i}}=$ acceleration of $P_{i}$ in the reference frame of the torso;

$404{ }^{N} \vec{\alpha}^{\mathrm{T}}=$ angular acceleration of the torso in the inertial frame.

Even though the central angular momentum is only changed by external torques, relative movements of body segments result in redistributions of angular momentum, which can change the orientation of the pigeon in 3D space, much like a cat righting itself in mid-air (see Frolich, 1980). Specifically, in the absence of aerodynamic forces, the external torque about $S_{\mathrm{CM}}$ and, thus, the rate of change of the central angular momentum are zero. Therefore, any changes in the 
411 angular momentum of the wings will be matched by an equal and opposite change in the angular

412 momentum of the rest of the bird (torso, head and tail), referred to here as the torso-system. By

413 equating the entire right-hand side of Eqn 1 to zero, the aerodynamic torque is assumed to be

414 zero, and ${ }^{N} \vec{\alpha}$ Torso can be solved for at each time step (see Appendix). By comparing the

415 expected angular velocity from inertia-based reorientations to the measured angular velocity of

416 the torso, torso angular accelerations based on aerodynamic forces can, in turn, be deduced.

417 Aerodynamics-based torso angular accelerations determined this way closely matched calculated

418 aerodynamic torques in timing and direction (not shown) and were used for further analysis, as

419 these torso angular accelerations could be directly compared with inertia-based torso angular

420 accelerations.

\section{Kinematics and aerodynamic torque generation}

423 Potential wing kinematic predictors of aerodynamic torques were assessed on an inside

424 wing vs outside wing basis. Accordingly, inside wing kinematics were averaged, as were outside

425 wing kinematics, for wingbeat pairs obtained from left turn and right turns. Due to the

426 incremental yet oscillatory nature of pigeon turning flight (Warrick et al., 1998), wingbeat

427 characteristics, such as variations in aerodynamic power output from one wingbeat to the next,

428 could lead to higher wing speeds or angles of attack of both the inside and outside wings in a left

429 turn as compared to a right turn (or vice versa), without accurately reflecting torque-related

430 contralateral asymmetries. The averaging of inside wing versus outside wing kinematics for

431 wingbeat pairs recorded across left and right turn flight trials accounted for such potential

432 differences between wingbeat cycles, as well as differential left wing - right wing marker 433 placement.

434 Even though active torque generation is required to change the orientation of a flying

435 bird, once a bird has reoriented in a particular way, say banked into a turn, even subsequent

436 wingbeats can change the flight direction, due to the previously obtained orientation. The

437 challenge was to identify wingbeats with active torque generation that were of similar dynamic

438 nature and of opposite sign, so that wingbeat pairs could be analyzed on the described inside

439 versus outside wing basis. 
Previously, we found that the occurrence of head saccades indicates wingbeats that contain active torque generation that lead to reorientations of the bird related to flight path

442 changes (Ros and Biewener, in review). In addition to the before-mentioned wingbeat-to-

443 wingbeat differences such as aerodynamic power and handedness, wingbeats can start with

444 existing angular momentum, further complicating the identification of wingbeats to select for the

445 analysis of torque generation. Eighteen key turning wingbeats (three left and three right per

446 individual) were selected from wingbeats with large head saccades, generally occurring early in

447 the turn, based on kinematic consistency in terms of torso angular velocity and acceleration

448 profiles (Fig. 5). The selection of these key turning wingbeats was not based on wing kinematics.

449 Consistent but opposing angular torso kinematics do not preclude differences in aerodynamic

450 flight power or negate handedness, still requiring an inside versus outside wing analysis of the

451 aerodynamic torque generating mechanism.

456 C.A. Moreno for helpful discussions and informal contributions to this work. We also thank two

457 anonymous reviewers for improving the manuscript. This study was funded by grants from NSF

458 (IOS-0744056) and ONR (N0014-10-1-0951) to AAB.

Author Contributions

IGR and AAB designed and executed the research. IGR, MAB and ANP analyzed the 462 data. All authors interpreted the results. IGR, AAB and LCB wrote the article.

\section{References}

465 1. Aldridge, H. D. J. N. (1986). Kinematics and aerodynamics of the Greater Horseshoe Bat 
3. Baier, D. B., Gatesy, S. M. and Dial, K. P. (2013). Three-Dimensional, High-Resolution Skeletal Kinematics of the Avian Wing and Shoulder during Ascending Flapping Flight

4. Berg, van den, C. and Rayner, J. M. V. (1995). The moment of inertia of bird wings and the inertial power requirement for flapping flight. J. Exp. Biol. 198, 1655-1664.

5. Bilo, D. (1994). Course control in flight. In M. N. O. Davies and P.R. Green (eds.), Perception and motor control in birds, pp. 227-247. Berlin: Springer-Verlag.

6. Bilo, D., Bilo, A., Muller, M., Theis, B. and Wedekind, F. (1985). Neurophysiologicalcybernetic analysis of course control in the pigeon. Biona Report 3, 445-477.

7. Dial, K. P. (1992). Avian forelimb muscles and nonsteady flight: Can birds fly without using the muscles in their wings? Auk 109, 874-885.

8. Dial, K. P. and Gatesy, S. M. (1993). Neuromuscular control and kinematics of the wings and tail during maneuvering flight. Am. Zool. 33, 5 .

9. Dial, K. P., Kaplan, S. R., Goslow, G. E., JR and Jenkins, F. A. JR (1988). A functional analysis of the primary upstroke and downstroke muscles in the domestic pigeon (Columba livia) during flight. J. Exp. Biol. 134, 1-16.

10. Dudley, R. (2002). Mechanisms and implications of animal flight maneuverability. Integr. Comp. Biol. 42, $135-140$.

11. Frohlich, C. (1980). Physics of somersaulting and twisting. Sci. Am. 242(3):155

12. Gatesy, S. M. and K. P. Dial. (1996). Tail muscle activity patterns in walking and flying pigeons (Columba livia). J. Exp. Biol. 176: 55-76.

13. Hedrick, T. L. and A. A. Biewener (2007). Low speed maneuvering flight of the rosebreasted cockatoo (Eolophus roseicapillus). I. Kinematic and neuromuscular control of turning. J. Exp. Biol. 210 (11), 1897-1911.

14. Hedrick, T. L., Cheng, B. and Deng, X. Y. (2009). Wingbeat time and the scaling of passive rotational damping in flapping flight. Science 324, 252-255.

15. Hedrick, T. L., Usherwood, J. R and Biewener, A. A. (2007). Low speed maneuvering flight of the rose-breasted cockatoo (Eolophus roseicapillus). II. Inertial and aerodynamic reorientation. J. Exp. Biol. 210 (11), 1912-1924. 
16. Hedrick, T.L. (2008). Software techniques for two- and three-dimensional kinematic measurements of biological and biomimetic systems. Bioinspir. Biomim. 3, 034001.

17. Iriarte-Diaz J. and Swartz S. M. (2008). Kinematics of slow turn maneuvering in the fruit bat Cynopterus brachyotis. J. Exp. Biol. 211:3478-3489.

18. Iriarte-Diaz J., Riskin D. K., Willis D. J., Breuer K. S. and Swartz S. M. (2011). Wholebody kinematics of a fruit bat reveal the influence of wing inertia on body accelerations. J. Exp. Biol. 214, 1546-1553.

19. Mitiguy, P. (2014). Dynamics of Mechanical, Aerospace, and Biomechanical Systems. Sunnyvale: Prodigy Press.

20. Newman, F. H. and Searle, V. H. L. (1957). The general properties of matter. London: Arnold.

21. Pratab, R. and Ruina, A. (2009). Introduction to Statics and Dynamics. Oxford: Oxford University Press.

22. Ros, I. G. (2013). Low Speed Avian Maneuvering Flight. Ph.D. Thesis. Harvard University.

23. Ros, I. G., Bassman, L. C., Badger, M. A., Pierson, A. N. and Biewener, A. A. (2011). Pigeons steer like helicopters and generate down- and upstroke lift during low speed turns. PNAS 108, 19990-19995.

24. Su, J. Y., Ting, S. C., Chang, Y. H., and Yang, J. T. (2011). Aerodynamic trick for visual stabilization during downstroke in a hovering bird. Phys. Rev. E 84, 012901.

25. Vazquez, R. J. (1995). Functional anatomy of the pigeon hand (Columba livia): a muscle stimulation study. J. Morph. 226(1), 33-45.

26. Walker, J. A. (1998). Estimating velocities and accelerations of animal locomotion: a

27. Warrick, D. R. (1998). The turning and linear maneuvering performance of birds: The cost of efficiency for coursing insectivores. Can. J. Zool. 76(6), 1063-1079.

524 28. Warrick, D. R. and Dial, K. P. (1998). Kinematic, aerodynamic, and anatomical mechanisms in the slow maneuvering flight of pigeons. J. Exp. Biol. 201, 655-672. the maneuvering flight of pigeons. Auk 115(4), 916-928. 
30. Winter, D. A. (2005). Biomechanics and Motor Control of Human Movement. Hoboken:

Wiley.

\section{Figure Captions}

533 Figure 1. Schematic top view of the flight corridor. Camera outlines represent viewing

534 angles, with camera distances underrepresented by $50 \%$, for the high-speed (blue) and infrared-

535 based auto-tracking (red) systems. The spatially calibrated section of the 90-degree turn (dark

536 blue), pigeon silhouettes, and perches (grey lines) are drawn to scale. Dimensions are noted

537 along the outside of one leg of the symmetrical corridor.

Figure 2. Dynamics model of a pigeon based on marker locations. (A) Cut-out of a pigeon at mid downstroke, copper-coloured for contrast, with marker locations (black circles with white centers). The torso and head (hd) (large and small grey ellipsoids) were treated as rigid objects

542 for which the moments of inertia were assigned about the principal torso axes (B): anterior-

543 posterior (roll, red line), medio-lateral (pitch, green line) and dorso-ventral (yaw, blue line). The

544 torso ellipsoid, which includes the tail, is positioned based the dorsal (dm) and rump (ru)

545 markers. Per wing, three articulating segments (solid green lines) are represented by point

546 masses: the brachium (one mass), antebrachium (two masses) and the hand wing distal to the

547 wrist (eleven masses). Respectively, these segments are reconstructed based on the shoulder (sh)

548 - elbow (el), elbow - wrist (wr) and wrist - center between the ninth (9p) and fifth (5p) primary

549 markers. Each pointmass represents a wingstrip (in between the dashed green lines). The

550 volumes of the spheres and ellipsoids represent relative masses. The dynamics model of a

551 pigeon in late downstroke (B), and near mid-upstroke (C).

553 Figure 3. Low-speed, level turns in pigeons are predominantly based on roll and yaw

554 rotations. Integrated angular velocities about the principal torso axes throughout a representative 555 right turn, with positive roll and yaw into the turn, as well as head-up pitch (small depictions

556 atop the figure). Initial angles are based on the inclinations of the corresponding torso axes with 557 respect to the horizontal at the start of recording. The majority of torso reorientations occurs 
558 about the roll and yaw axes. Only pitch varies periodically with the wingbeat (blue shading 559 represents downstroke phases). Flight speed (grey trace), a one-wingbeat running average, remains approximately constant with a moderate slow down near the midpoint of the turn $(\mathrm{t}=0$

561 s).

Figure 4. Aerodynamic estimates predict torso angular accelerations. Torso angular

564 accelerations based on aerodynamic torque estimates (“Aerodynamics"; left panels) predict

565 measured torso accelerations throughout two left and two right turns per individual (N=3; plotted symbols), about roll (dark/red, top left; $\mathrm{R}^{2}=0.72$, $\mathrm{p}<0.01$ ), pitch (medium/green, center left; $\left.\mathrm{R}^{2}=0.89, \mathrm{p}<0.01\right)$ and yaw (light/blue, bottom left; $\left.\mathrm{R}^{2}=0.62, \mathrm{p}<0.01\right)$ axes. Torso angular accelerations based on rate of momentum exchange ("Inertia"; right panels) between the wings and the torso do not correlate with observed roll and pitch torso accelerations, but do correlate with observed yaw accelerations (light/blue, bottom right; $\mathrm{R}^{2}=0.41, \mathrm{p}<0.01$ ). The regression analyses about the principal torso axes are based on one (peak) torso acceleration per wingbeat and corrected for trial and wingbeat numbers and individual effects.

573 Figure 5. Roll velocity is initiated early and arrested late in the downstroke. (A) Mean \pm sd 574 of individual mean ( $\mathrm{N}=3$ ) torso velocities in roll (red), pitch (green) and yaw (blue) throughout 575 three left and three right selected wingbeats early in the turn, and normalized to the wingbeat

576 period. Near the middle of the downstroke (blue shading) the torso pitches bill-down (-) and rolls 577 into the turn (+). (B) The mean individual roll and pitch accelerations peak in the first half of the 578 downstroke (red and green, respectively), with extremes reversed in the second half of the 579 downstroke, arresting the roll velocity and reversing the pitch velocities in (A), respectively.

580 Pitch accelerations also show peaks near mid-upstroke, whereas roll accelerations are more 581 variable during the upstroke.

583 Figure 6. Turning wingbeats are characterized by differentially swept wing trajectories.

584 Mean \pm sd (trace thickness) of individual mean trajectories of the distal wing marker for the 585 inside (green) and outside (purple) wing for the same selected turning wingbeats as in Fig. 5. 586 Both trajectories are represented as a right wing, by mirroring the left wing in the mid-sagittal 587 plane. The trajectories are displayed relative to the torso frame, represented by the anterior (red), 
right (green) and dorsal (blue) directions, as well as the right shoulder location (black sphere).

589 The means of the 3D trajectories, with the upstroke in a lighter shade, are projected on each of

590 the anatomical planes, emphasizing the more protracted (anteriorly projected) path of the outside

591 wing early in the downstroke.

Figure 7. Wing pronation accompanies a more caudally swept inside wing, whereas a

594 greater wing speed and later projection aerodynamically dampen roll velocity. (A) For the

595 same wingbeats as in Fig. 5; 6, during the upstroke, the inside wing (green) and outside wing

596 (purple) supinate and subsequently pronate about the wing long-axis in synchrony. Early in the

597 downstroke, the inside wing pronates to a larger degree than the outside wing (purple), resulting

598 in an inside versus outside wing differential that is negative, or pronated (black trace \pm sd). The

599 more pronated inside wing in combination with the contra-lateral difference in wing trajectory

600 maintains comparable angles of attack between the wings. However, these wing-configuration

601 and kinematic differences likely results in aerodynamic forces that act above the center of mass

602 to be directed upwards and to the right side of the torso, causing the pitch down and roll torques

603 into the turn. (B) Inside wing speed (of the ninth primary marker) starts surpassing outside wing

604 speed as the roll velocity (A) increases towards the inside wing. The higher speed of the inside

605 wing persists longer than the roll velocity, indicating its contribution to passive aerodynamic roll

606 damping, aided by a more lateral orientation angle $(\mathrm{C})$ of the inside wing (the angle between the

607 wing long axis and the sagittal plane of the torso).

609 Figure 8. Interpretation of aerodynamic pitch and roll torques. Aerodynamic pitch torques,

610 (A) normalized to the wingbeat cycle, of a representative individual (mean \pm sd of 20 turning

611 wingbeats) were (B) consistent across individuals (mean \pm sd of individual means $(\mathrm{N}=3)$ ).

612 Negative (head-down) aerodynamic pitch torques early in downstroke and at the downstroke-

613 upstroke transition are offset by positive peaks in pitch torque in the latter halves of both the

614 upstroke and downstroke. These patterns, combined with wing stroke kinematics (Figs. 6 \& 7),

615 indicate that the resultant aerodynamic force acts behind $S_{\mathrm{CM}}$ (black-white symbol in C and D)

616 during the early part of the downstroke and subsequently travels antero-ventrally to produce an

617 opposing head-up torque by acting in front of $S_{\mathrm{CM}}$ later in downstroke. (C, D) Interpretation of 
618 roll-in (C) and pitch-down (D) torque generating mechanisms. Rear-view of a cut-out of a pigeon 619 entering a left turn, early in the downstroke, with superimposed anatomical roll (AP; red), pitch

620 (ML; green) and yaw (DV; blue) axes (see Fig. 2B). (C) An approximation of the instantaneous

$6213 \mathrm{D}$ resultant aerodynamic force (black vector, $\mathrm{F}_{\text {aero }}$ ) at this moment of the wingbeat cycle is

622 projected onto the transverse ML-DV plane (teal dashed vector within the teal shading). The line

623 of action (teal dotted line) of this transverse projection extends to the right of $S_{\mathrm{CM}}$, with a

624 moment arm (solid teal line) to produce a left roll torque into the turn (red circular arrow). A

625 more pronated left wing and a more anteriorly swept right wing result in an aerodynamic force

626 that is directed left of the mid-sagittal plane. (D) The 3D resultant aerodynamic force, $\mathrm{F}_{\text {aero, }}$

627 projected onto the mid-sagittal AP-DV plane (purple dashed vector within purple shading) acts,

628 similarly, behind $S_{\mathrm{CM}}$ to produce a head-down pitch torque (green circular arrow). The picture

629 was taken of a turning pigeon without markers. 


\section{Tables}

632

633 Table 1. Morphological inertia properties measured for Columba livia $(\mathrm{N}=3)$ : Segment masses.

\begin{tabular}{lr}
\hline Segment & \% of BM \\
\hline Torso (including tail) & $83.6 \pm 1.3$ \\
Head & $3.9 \pm 0.3$ \\
Brachium & $1.5 \pm 0.3$ \\
Ante-brachium & $2.9 \pm 0.4$ \\
Hand wing & $1.9 \pm 0.1$ \\
\hline
\end{tabular}

635 Table 2. Net wingbeat reorientations accumulated throughout the turn ( $\mathrm{N}=3$; individual means).

\begin{tabular}{ll}
\hline $\begin{array}{l}\text { Torso rotation } \\
\text { components }\end{array}$ & $\begin{array}{l}\text { Reorientation }(\mathrm{deg}) \\
(\mathrm{mean} \pm \mathrm{sd})\end{array}$ \\
\hline Roll & $76.8 \pm 13.6$ \\
Pitch & $42.6 \pm 1.6$ \\
Yaw & $58.1 \pm 4.3$ \\
\hline
\end{tabular}

638 Table 3. Morphological inertia properties measured for Columba livia $(\mathrm{N}=3)$ : Mass moments of 639 inertia $($ mean \pm sd).

640

\begin{tabular}{lrrr}
\hline$\left(* 10^{-5} \mathrm{~kg}^{*} \mathrm{~m}^{2}\right)$ & \multicolumn{1}{l}{ Roll } & \multicolumn{1}{l}{ Pitch } & \multicolumn{1}{l}{ Yaw } \\
\hline Torso & $20.98 \pm 3.75$ & $56.38 \pm 0.28$ & $71.19 \pm 8.62$ \\
Head & $3.90 \pm 0.45$ & $5.93 \pm 0.90$ & $4.42 \pm 0.13$ \\
\hline
\end{tabular}


A

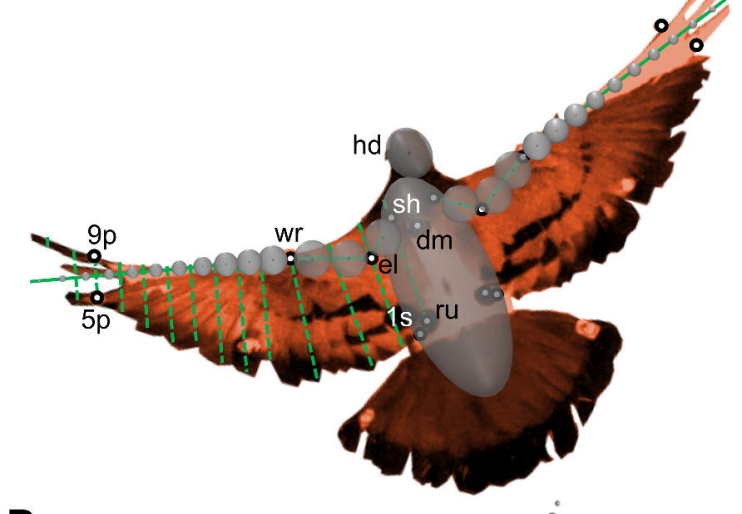

- 00000

B

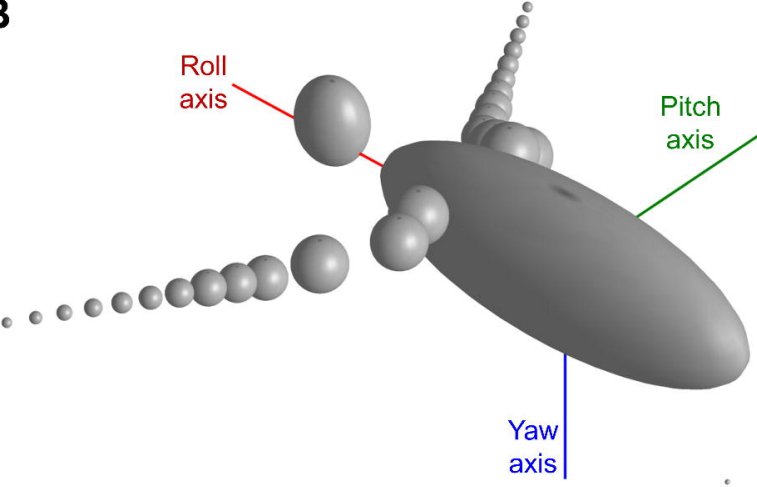

C

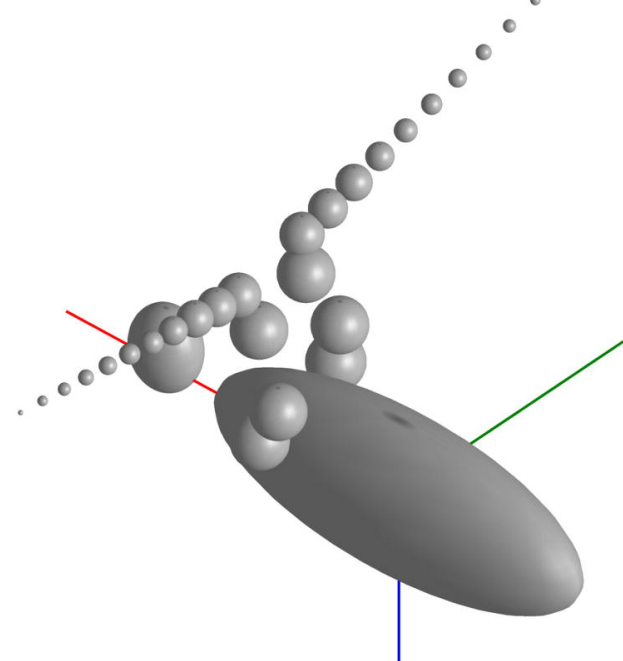


+ Roll + Pitch + Yaw

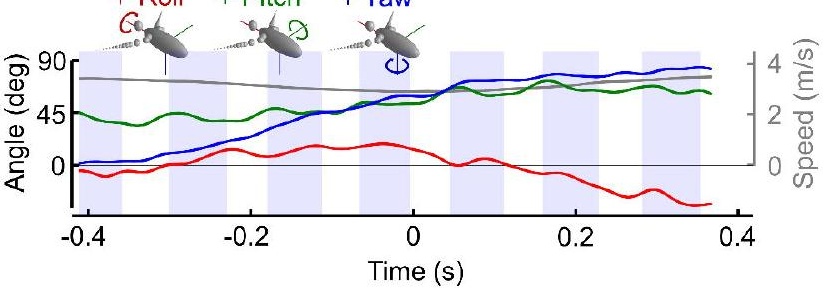





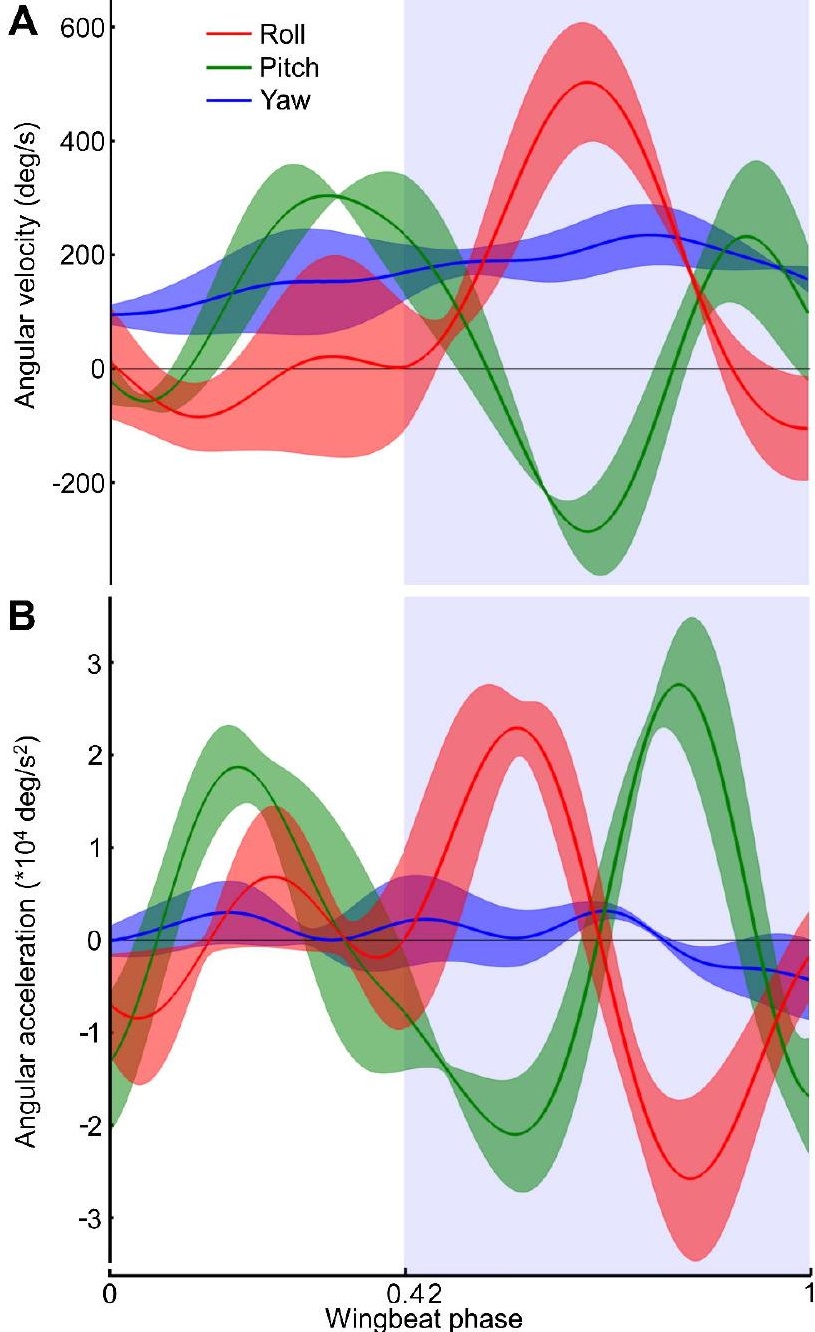




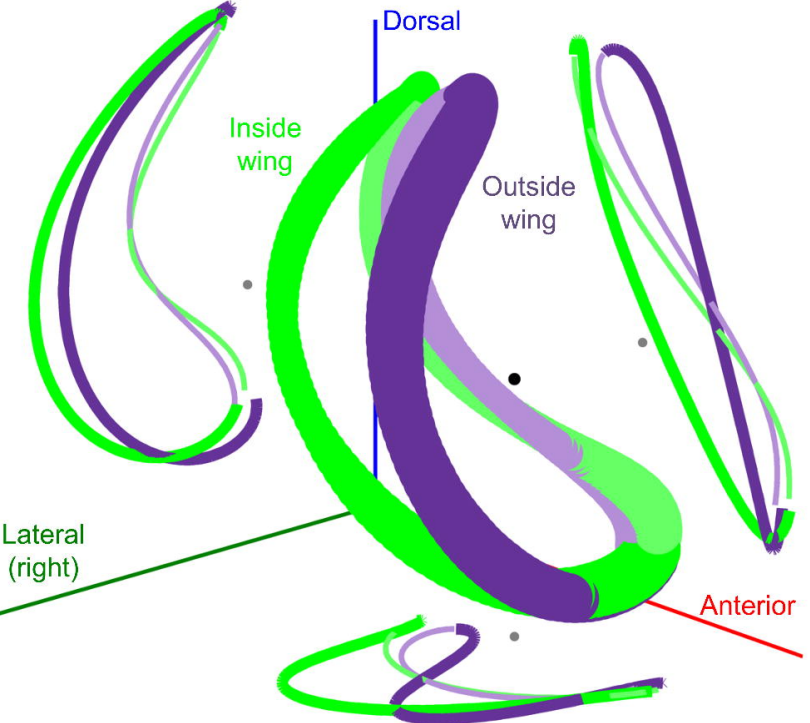



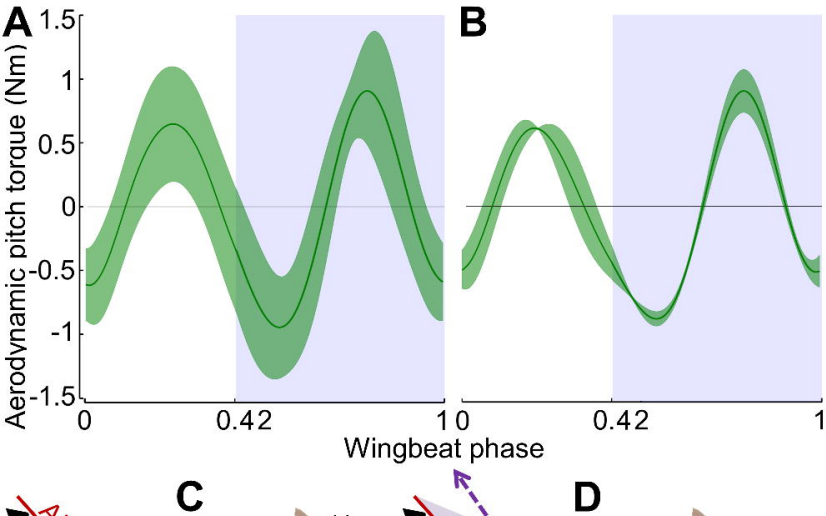

No
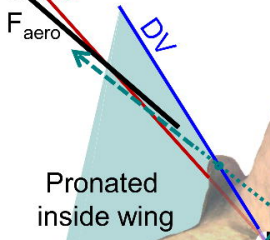

inside wing

is
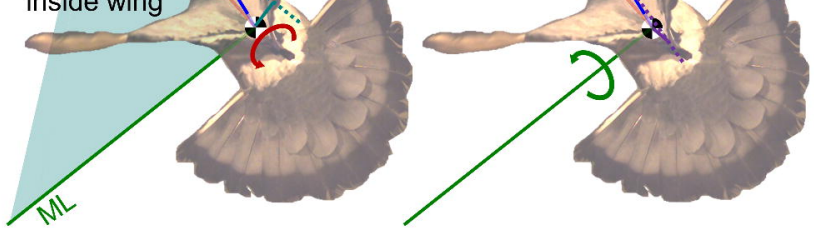